https://helda.helsinki.fi

\title{
Association of BMI-1 and p16 as prognostic factors for head and neck carcinomas
}

\section{Lundberg, Marie}

2016-05-03

Lundberg, M , Renkonen , S, Haglund, C , Mattila, P S , Leivo , I , Hagstrom , J \& Makitie , A A 2016 , ' Association of BMI-1 and p16 as prognostic factors for head and neck carcinomas ' , Acta Oto-Laryngologica , vol. 136 , no. 5 , pp. 501-505 . https://doi.org/10.3109/00016489.2015.11222

http://hdl.handle.net/10138/161459

https://doi.org/10.3109/00016489.2015.1122227

publishedVersion

Downloaded from Helda, University of Helsinki institutional repository.

This is an electronic reprint of the original article.

This reprint may differ from the original in pagination and typographic detail.

Please cite the original version. 


\section{Association of BMI-1 and p16 as prognostic factors for head and neck carcinomas}

\section{Marie Lundberg, Suvi Renkonen, Caj Haglund, Petri S Mattila, Ilmo Leivo, Jaana Hagström \& Antti A Mäkitie}

To cite this article: Marie Lundberg, Suvi Renkonen, Caj Haglund, Petri S Mattila, Ilmo Leivo, Jaana Hagström \& Antti A Mäkitie (2016) Association of BMI-1 and p16 as prognostic factors for head and neck carcinomas, Acta Oto-Laryngologica, 136:5, 501-505, DOI: 10.3109/00016489.2015.1122227

To link to this article: http://dx.doi.org/10.3109/00016489.2015.1122227

册Published online: 12 Jan 2016.

Submit your article to this journal ¿

Џ Article views: 67

Q View related articles $₫$

View Crossmark data $\asymp$ 


\title{
Association of BMI-1 and p16 as prognostic factors for head and neck carcinomas
}

\author{
Marie Lundberg ${ }^{a *}$, Suvi Renkonen ${ }^{a, b *}$, Caj Haglund ${ }^{c, d, e}$, Petri S Mattila ${ }^{a}$, Ilmo Leivo ${ }^{f}$, Jaana Hagström ${ }^{c}$ \\ and Antti A Mäkitie $e^{a, b}$ \\ a Department of Otorhinolaryngology - Head and Neck Surgery, University of Helsinki and Helsinki University Hospital, Helsinki, Finland; \\ ${ }^{\mathrm{b}}$ Division of Ear, Nose and Throat Diseases, Department of Clinical Sciences, Intervention and Technology, Karolinska Institutet and Karolinska \\ Hospital, Stockholm, Sweden; 'Department of Pathology, Haartman Institute and HusLab, University of Helsinki and Helsinki University Hospital, \\ Helsinki, Finland; ${ }^{d}$ Department of Surgery, University of Helsinki and Helsinki University Hospital, Helsinki, Finland; ${ }^{e}$ Research Programs Unit, \\ Translational Cancer Biology, University of Helsinki, Helsinki, Finland; ' Department of Pathology, Turku University Hospital and University of \\ Turku, Turku, Finland
}

\begin{abstract}
Conclusions BMI-1 is an upstream repressor of tumor suppressor p16 and their inverse expression patterns have been linked with patient survival in OPSCC. In this material only p16 remained a relevant prognostic marker in OPSCC.

Objectives HNSCC tumors carry variable phenotypes and clinical outcomes depending on their anatomical location. In OPSCC, expression of tumor suppressor p16 is used as a surrogate marker of HPV infection and has prognostic value. There are no good prognostic biomarkers for HNSCC tumors of other anatomical locations.

Aim To study the expression patterns of p16 and BMI-1 in not only oropharyngeal but also oral, hypopharyngeal, and laryngeal squamous cell carcinomas and to clarify their putative connections with clinical parameters, survival, and each other.

Method Hospital records on 130 patients (59 OPSCC, 18 OSCC, 20 HPSCC, and 33 LSCC) diagnosed between 1997-2008 at the Helsinki University Hospital, Finland, were reviewed. BMI-1 and p16 expressions were studied by immunohistochemistry.

Results Sixty-eight per cent of OPSCC expressed p16 and expression correlated with lower age, lower T- and higher N-category, and with improved OS and DFS. BMI-1 expression was most prevalent in OPSCC and LSCC, but had no clinical correlations. No correlation between p16 and BMI-1 expression was found.
\end{abstract}

\section{ARTICLE HISTORY}

Received 2 September 2015

Revised 1 November 2015

Accepted 10 November 2015

Published online

24 December 2015

\section{KEYWORDS}

Oral; oropharynx;

hypopharynx; larynx; stem cell; predictive marker; HPV

\section{Introduction}

The 5-year survival in head and neck squamous cell cancers (HNSCC) varies according to tumor anatomical location and stage, and even within clinically homologous tumors treatment response can be unpredictable. Numerous studies have tried to find biomarkers that would foresee tumor behavior and aid in clinical decision-making. Currently, the most reliable biomarkers in use are the presence of human papilloma virus (HPV) and the expression of its surrogate marker p16 in oropharyngeal squamous cell carcinoma (OPSCC) - HPV being the only prognostic marker cited in the 2015 NCCN guidelines [1-3]. For HNSCC of other anatomical localizations there are still no good biomarkers in clinical use.

It is thought that the malignant transformation of HPV positive OPSCC tumor is mainly caused by oncoproteins E6 and E7 [1,2]. The unspliced variant of E6 oncoprotein forms a complex with an ubiquitin-protein ligase leading to subsequent degradation of tumor suppressor p53 through its ubiquitination $[1,2]$. E7 oncoproteins inactivate another important tumor suppressor $\mathrm{Rb}$ and its associated pocket proteins. This inactivation leads to over-expression of active E2F transcription factors resulting in increased cell proliferation $[1,2]$. Due to a negative feedback loop, $\mathrm{Rb}$ inactivation in HPV positive tumors leads to increased levels of p16 [1-3]. This is why immunohistochemical staining of p16 protein expression can be used as a biomarker for tumor infected with HPV virus [3]. p16 is a tumor suppressor encoded by the INK4a/Arf locus. p16 inhibits cyclin D1-cyclin dependent kinase complex that acts through phosphorylation of tumor suppressor $\mathrm{Rb}$ [4]. After phosphorylation, $\mathrm{Rb}$ protein becomes inactive, which enables the cell cycle to progress and tumor growth. As p16 inhibits the inactivation of $\mathrm{Rb}$, high levels of p16 lead to cell cycle arrest [1].

B-cell-specific Moloney murine leukemia virus integration site 1 (BMI-1) is a transcription factor and epigenetic regulator, essential in maintaining the transcriptionally repressed state of many genes through methylation and acetylation of chromatin and histones [5]. BMI-1 regulates genes involved in the cell cycle and cell differentiation and can, therefore, act as a potent oncogene [6,7]. BMI-1's effect is mediated partly through repression of the INK4a/ARF, a locus encoding p16 [4]. Upregulation of BMI-1 leads to repression of p16. This causes 
tumor suppressor $\mathrm{pRb}$ inactivation through phosphorylation, leading to cell cycle progression. Over-expression of BMI-1 has been shown in multiple malignant tumors, including nasopharyngeal and oral carcinomas [6,8]. In HNSCC, BMI-1 expression is linked with promotion of both tumor formation and invasion, as well as tumors metastatic capacity and increased resistance to ionizing radiation $[6,7,9,10]$.

In OPSCC, p16 has been associated with better survival [3] The presence and significance of this association in other types of HNSCC remains unclear [11,12]. BMI-1 expression has been linked with both better and worse survival of cancer patients. In OPSCC and laryngeal squamous cell carcinoma an inverse relation between p16 and BMI-1 expression has been reported to affect tumor stage and patient survival $[4,13]$. In this study we investigated the expression of p16 and BMI-1 in OPSCC, oral (OSCC), hypopharyngeal (HPSCC), and laryngeal (LSCC) squamous cell carcinoma. Our aim was to clarify the possible associations between expression levels of these two proteins and to assess their use as putative prognostic factors of survival in different types of HNSCC.

\section{Patients and methods}

Retrospective clinicopathological data of 130 patients diagnosed with histologically verified HNSCC between 1997-2008 at the Helsinki University Hospital, Helsinki, Finland were reviewed. There were 59 OPSCC patients, 33 LSCC, 20 HPSCC, and 18 OSCC. Patients with nasopharyngeal tumors were excluded, as were patients with unknown primaries, and patients with less than 2 years follow-up time. All patients were treated with curative intent. Patient characteristics are shown in Table 1.

For immunohistochemical (IHC) stainings we used 130 formalin-fixed and paraffin-embedded tumor samples obtained from the archives of Department of Pathology, Helsinki University Hospital. The samples were cut into 4-5 $\mu \mathrm{m}$-thick sections, deparaffinized in xylene, and rehydrated through a graded alcohol series. For antigen retrieval, slides were treated in a PT module (LabVision, Cambridgeshire, UK) with Tris-HCl buffer (8.5) (for BMI-1) or Tris-EDTA (9.0) for p16. BMI-1 IHC was performed in

Table 1. Patient characteristics of 130 HNSCC patients.

\begin{tabular}{llclll}
\hline \multicolumn{1}{c}{} & & OSCC & \multicolumn{1}{c}{ OPSCC } & \multicolumn{1}{c}{ HPSCC } & \multicolumn{1}{c}{ LSCC } \\
& & $n=18(\%)$ & $n=59(\%)$ & $n=20(\%)$ & $n=33(\%)$ \\
\hline Sex & Men & $12(67)$ & $40(68)$ & $18(90)$ & $30(91)$ \\
& Women & $6(33)$ & $19(32)$ & $2(10)$ & $3(9)$ \\
Mean age (range) & $57(26-79)$ & $56(29-73)$ & $58(31-81)$ & $61(40-84)$ \\
T category & $1-2$ & $11(61)$ & $40(68)$ & $4(20)$ & $13(40)$ \\
& $3-4$ & $7(39)$ & $29(32)$ & $15(75)$ & $20(60)$ \\
N category & $0-1$ & $15(84)$ & $25(42)$ & $7(35)$ & $28(85)$ \\
\multirow{5}{*}{ Stage } & 2-3 & $3(16)$ & $34(58)$ & $13(65)$ & $4(12)$ \\
& I-II & $9(50)$ & $8(14)$ & $0(0)$ & $11(33)$ \\
Treatment & III-IV & $9(50)$ & $51(86)$ & $20(100)$ & $22(66)$ \\
& Surgery & $8(44)$ & $1(2)$ & $0(0)$ & $6(18)$ \\
& RT & $0(0)$ & $0(0)$ & $0(0)$ & $3(9)$ \\
& CRT & $0(0)$ & $5(9)$ & $14(70)$ & $7(21)$ \\
BMI-1 & Combined & $10(56)$ & $53(90)$ & $6(30)$ & $17(52)$ \\
& Negative & $14(78)$ & $25(42)$ & $14(70)$ & $20(61)$ \\
& Positive & $4(22)$ & $34(58)$ & $6(30)$ & $13(39)$ \\
& Low & $3(17)$ & $28(48)$ & $3(15)$ & $13(39)$ \\
p16 & High & $1(5)$ & $6(10)$ & $3(15)$ & $0(0)$ \\
& Negative & $17(94)$ & $19(32)$ & $16(80)$ & $32(97)$ \\
& Positive & $1(6)$ & $40(68)$ & $4(20)$ & $1(3)$ \\
\hline
\end{tabular}

Autostainer 480 (LabVision). Slides were treated with $0.3 \%$ Dako REAL peroxidase-blocking solution to block endogenous peroxidase activity followed by primary antibody incubation with mouse monoclonal BMI-1 (ab 14389) (Abcam, Cambridge, UK, 1:750) or ready-to-use mouse anti-human p16 INK4a antibody clone $9511 \mathrm{CINtec}^{\mathrm{TM}}$ histology kit (MTMLabs, Heidelberg, Germany), followed by a 30-min incubation with Dako REAL EnVision/HRP detection system, rabbit/mouse (ENV) reagent. Ten minutes of Dako REAL $\mathrm{DAB}+$ Chromogen (Dako) finally visualized reaction products. PBS-0.04\%-Tween 20 washing was accomplished between each step. Slides were counterstained with Meyer's hematoxylin and mounted in mounting medium (Aquamount, BDH, Poole, UK). The BMI-1 antibody has previously been used in other published studies from our department [14] and p16 is in routine diagnostic use at the Department of Pathology.

IHCs were evaluated by two independent pathologists (IL and $\mathrm{JH}$ ), blinded to clinical data. They approximated the percentage of positive cells within the tumor tissue. If $70 \%$ or more of the tumor cells had nuclear and cytoplasmic staining of p16, the expression was classified as positive (Figure 1). BMI-1 staining was originally classified into six categories: negative, extremely low ( $1-5 \%$ of tumor nuclei stained), very low (6-30\%), low (31-50\%), moderate (51-80\%), and high $(>80 \%)$, but because of the small patient numbers in each category, the groups were merged according to Hayry et al. [14] into negative, low (1-50\%), and high expression $(\geq 51 \%$, Figure 1).

Statistical analysis was performed with IBM SPSS statistics version 22. Anatomical sub-groups were analyzed separately. Contingency variables were analyzed with Chi-square test or Fischer's exact test, means compared with Student's $t$-test and correlations with bivariate Pearson's score. For survival calculations we used Kaplan-Meier log-rank test and Cox regression analysis where patient's gender, age, stage, and molecular markers were included. A $p$ value of 0.05 was considered statistically significant.

\section{Results}

\section{Patients}

The vast majority of the patients were men (79\%), the percentage being extremely high in HPSCC (90\%) and LSCC (91\%). The mean age ranged from 56-61 years, being lowest in patients with OPSCC and OSCC. More specific patient characteristics and treatment modalities are described in Table 1.

\section{BMI-1 expression}

The intensity of BMI-1 expression varied from negative to high. Only nuclear expression pattern was regarded as positive. In OSCC and HPSCC, most samples were BMI-1 negative, whereas in LSCC $39 \%$, and in OPSCC $58 \%$ of the samples expressed nuclear BMI-1 but the differences were insignificant. Only 10 of the 130 tumors showed high levels of BMI-1 expression (Table 1). 

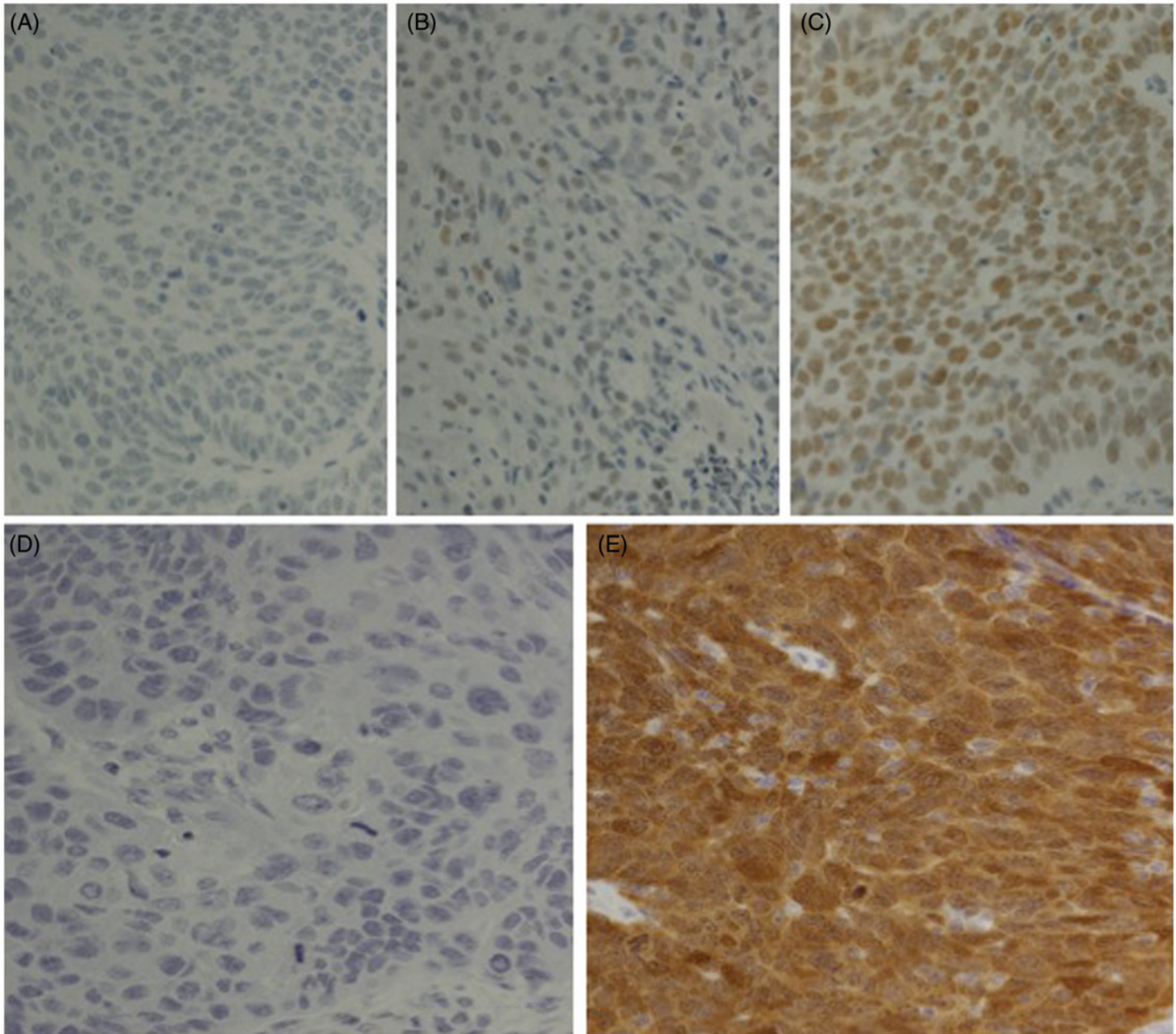

Figure 1. Immunohistochemical stainings of $B M I-1 \quad(A=$ negative, $B=$ low staining, $1-50 \%$, and $C=$ high staining, $\geq 51 \%)$, and p16 $(D=$ negative, and $E=$ positive, $\geq 70 \%$ of cells stained).

\section{BMI-1's correlations with clinical parameters}

BMI-1 expression had no correlation with clinical parameters including mean age, tumor size, nodal status, or stage in any anatomical sub-groups.

\section{p16 expression}

When present, p16 expression was intense and uniform. Positive immunostaining was most common in OPSCC (68\%), whereas in OSCC, HPSCC, and LSCC it was sparse $(6 \%, 20 \%$, and $3 \%$, respectively).

\section{p16's correlations with clinical parameters}

In OPSCC, patients with p16 positive tumors were on average younger than those with p16 negative tumors (54 vs 60 years, $p=0.007)$. The same tendency was also seen in HPSCC, although this finding lacked statistical significance (53 vs 60 years, $p=0.31)$. In OPSCC, 16 positivity was associated with lower T category $(p=0.004)$ and higher $\mathrm{N}$ category $(p<0.001)$, an association not seen in other groups. Statistical analysis was not possible for OSCC and LSCC due to the small number of patients (Table 1).

\section{Survival}

In OPSCC, positive p16 expression was linked with markedly improved overall survival (OS, $\mathrm{HR}=0.27,95 \% \mathrm{CI}=0.07-1.00$, $p=0.05$ ) and disease-free survival (DFS, HR $=0.27,95 \%$ $\mathrm{CI}=0.09-0.83, p=0.02$, Figure 2 ), and this result remained significant in multivariate analysis. A slight tendency towards improved survival in BMI-1 negative OPSCC patients could be seen, but this was statistically non-significant. When expression of BMI-1 and p16 were combined in survival analysis, it was clear that the effect of p16 outweighed that of BMI-1 (Figure 3).

In OSCC, BMI-1 expression, which was positive in only four patients, seemed to be associated with decreased survival in DFS $(p<0.001$, Figure 4$)$, and the result was confirmed in multivariate survival $(\mathrm{HR}=5.03 \quad 95 \%$ $\mathrm{CI}=1.20-20.91, p=0.03)$. In HPSCC or LSCC no statistically significant effects of p16 or BMI-1 expression on survival were seen.

\section{BMI-1 and p16 correlations}

We found no significant correlations between p16 and BMI-1 expression in any anatomical locations. 


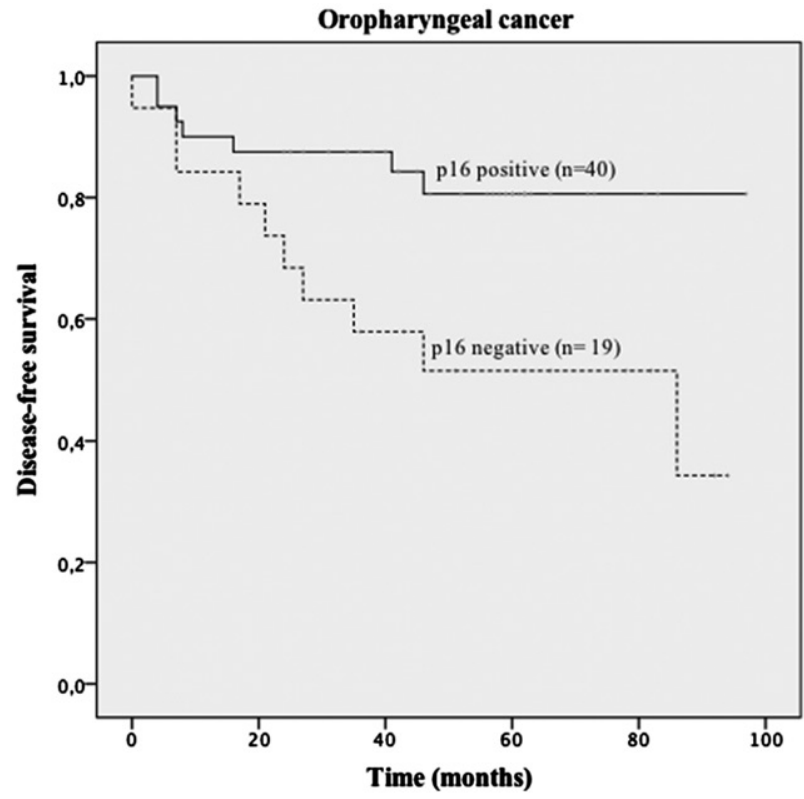

Figure 2. Kaplan-Meier survival curve of p16 expression in oropharyngeal tumors, $p=0.018$.

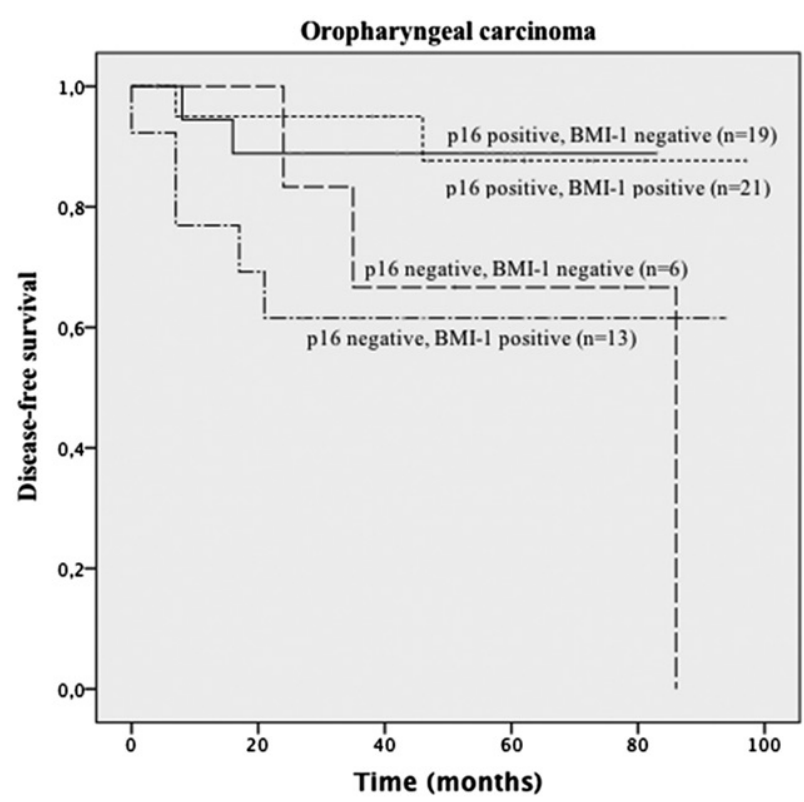

Figure 3. When combining $\mathrm{p} 16$ and BMI-1 expression in oropharyngeal tumors, p16 expression seemed to have a greater impact on survival $(p=0.11)$.

\section{Discussion}

BMI-1 is a member of a family of transcriptional repressors [15] and a known upstream modulator of p16 [16]. BMI-1's effect on p16 expression is of interest as p16 is widely in clinical use as a surrogate marker of HPV infection in OPSCC [3]. Elevated levels of BMI-1 have been reported in several cancers and its over-expression has been linked with cancer therapy failure. However, in tongue cancer, lack of expression has been shown to be associated with recurrence [14]. This contradiction could be explained by the fact that, in addition to its repressive effect on tumor suppressor p16, BMI-1 acts also

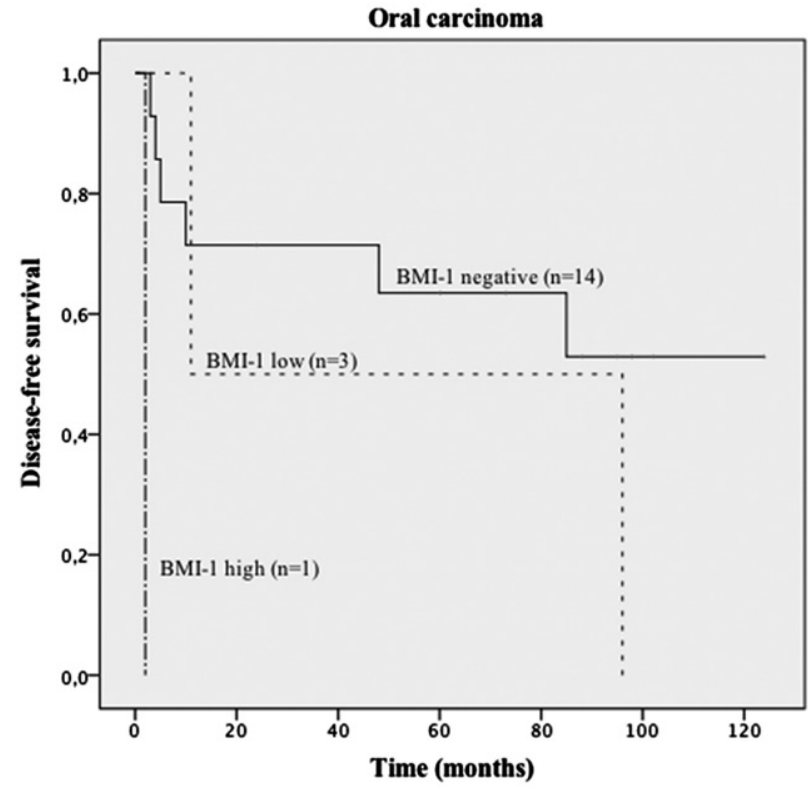

Figure 4. Disease-free survival curve of BMl-1 expression in oral carcinoma patients $(n=18), p<0.001$

via numerous other pathways independent of p16. It has also been suggested that BMI-1 alone should not be sufficient for tumor progression $[8,16]$.

Squamous cell carcinomas of the head and neck area are a heterogeneous group of tumors with different phenotypes and clinical outcomes. Several oncogenes/-proteins and tumor suppressors have been studied as they are thought to form the basis of biological tumor behavior, and their different expression levels might serve as predictive or prognostic factors. In this study, we investigated the expression levels of both p16 and BMI-1 in HNSCC of different anatomical locations, in order to study their co-expression's possible effect, as well as their associations with clinical parameters, and survival. In OPSCC, p16 expression was associated with better OS and DFS, and p16 positivity was linked with younger age, low $\mathrm{T}$ and high $\mathrm{N}$ categories - as was expected [17]. In other HNSCC tumors, p16 expression was low $(\mathrm{OSCC}=6 \%$, HPSCC $=20 \%, \quad$ LSCC $=3 \%$ ) and had no clear correlation with clinical parameters or survival. This finding was in good concordance with earlier results reporting up to $20 \%$ of nonOPSCC patients to have p16 positive tumors $[11,18]$.

BMI-1 is expressed in the cells of healthy oral mucosa and also in tumor cells of various HNSCC [4,6-9,13,14]. Previously both nuclear and cytoplasmic BMI-1 expressions have been scored $[4,7,13]$. In our study, we scored the samples only for nuclear staining, and used a cut-off of $50 \%$ for positive staining - a system previously used in our institution [14]. The cut-off points in other studies range from dichotomic positivevs-negative to $50 \%[6,9,14]$. In this cohort the expression of BMI-1 was more prevalent in OPSCC than in OSCC and HPSCC. The expression in LSCC (39\%) was in line with earlier reports of $44-50 \%[7,13]$. BMI-1 expression had no significant correlation with clinical parameters. Based on positive BMI-1 staining of four patients with OSCC, we found an association between the absence of BMI-1 expression and improved survival in OSCC. A similar, statistically non-significant trend 
was also seen in OPSCC. These findings are in line with earlier reports on OPSCC, LSCC and nasopharyngeal carcinoma $[4,6,7,13]$, but reciprocal when compared with the results of Hayry et al. [14] on 73 patients with T1-T2N0 tongue cancers. It is clear that further studies on larger patient cohorts are needed before this finding can be further discussed.

As BMI-1 should repress p16 expression via the INK4a locus [19], it is interesting to investigate the possible linkage between their expression levels. Huber et al. [4] were able to show that negative p16 expression, together with high cytoplasmic BMI-1 expression, is associated with poor survival in OPSCC. In LSCC nuclear co-expression, has been linked with a higher risk for lymph node metastasis [13]. In our limited material, we were not able to show a correlation between p16 and BMI-1 expression. It is possible that p16 was regulated through another pathway than that by BMI-1. Whether this could be explained for example by the presence of HPV remains unclear, as HPV status of the tumors was not investigated in our patients.

We conclude that our finding of p16 expressions correlation to younger age, small primary tumor with early regional spread and to better overall and disease-free survival in patients with OPSCC, is convergent with current literature $[1,17,18]$. BMI-1 positivity was most common in patients with OPSCC and had no clear correlation with clinical parameters. A trend towards better survival in BMI-1 negative patients was seen in OPSCC and OSCC. Although BMI-1 is known to be an upstream repressor of p16 and the expression levels of these two markers have previously been linked with each other this phenomenon was not seen in the present material. Whether this will be explained by an alternative, p16-regulating pathway, is warrant for further studies.

\section{Declaration of interest}

The authors report no conflicts of interest. The authors alone are responsible for the content and writing of the paper.

\section{References}

1. Rautava J, Syrjanen S. Biology of human papillomavirus infections in head and neck carcinogenesis. Head Neck Pathol 2012;6:S3-15.

2. Bol V, Gregoire V. Biological basis for increased sensitivity to radiation therapy in HPV-positive head and neck cancers. Biomed Res Int 2014;2014:696028.

3. Gronhoj Larsen C, Gyldenlove M, Jensen DH, Therkildsen MH, Kiss K, Norrild B, et al. Correlation between human papillomavirus and p16 overexpression in oropharyngeal tumours: a systematic review. Br J Cancer 2014;110:1587-94.
4. Huber GF, Albinger-Hegyi A, Soltermann A, Roessle M, Graf N, Haerle SK, et al. Expression patterns of Bmi-1 and p16 significantly correlate with overall, disease-specific, and recurrence-free survival in oropharyngeal squamous cell carcinoma. Cancer 2011;117:4659-70.

5. Spivakov M, Fisher AG. Epigenetic signatures of stem-cell identity. Nat Rev Genet 2007;8:263-71.

6. Song LB, Zeng MS, Liao WT, Zhang L, Mo HY, Liu WL, et al. Bmi-1 is a novel molecular marker of nasopharyngeal carcinoma progression and immortalizes primary human nasopharyngeal epithelial cells. Cancer Res 2006;66:6225-32.

7. Allegra E, Puzzo L, Zuccala V, Trapasso S, Vasquez E, Garozzo A, et al. Nuclear BMI-1 expression in laryngeal carcinoma correlates with lymph node pathological status. World J Surg Oncol 2012;10:206.

8. Kang MK, Kim RH, Kim SJ, Yip FK, Shin KH, Dimri GP, et al. Elevated Bmi-1 expression is associated with dysplastic cell transformation during oral carcinogenesis and is required for cancer cell replication and survival. Br J Cancer 2007;96:126-33.

9. Vormittag L, Thurnher D, Geleff S, Pammer J, Heiduschka G, Brunner $\mathrm{M}$, et al. Co-expression of Bmi-1 and podoplanin predicts overall survival in patients with squamous cell carcinoma of the head and neck treated with radio(chemo)therapy. Int J Radiat Oncol Biol Phys 2009;73:913-18.

10. Chen H, Zhou L, Wan G, Dou T, Tian J. BMI1 promotes the progression of laryngeal squamous cell carcinoma. Oral Oncol 2011;47:472-81.

11. Hoffmann M, Tribius S, Quabius ES, Henry H, Pfannenschmidt S, Burkhardt C, et al. HPV DNA, E6*I-mRNA expression and p16INK4A immunohistochemistry in head and neck cancer how valid is p16INK4A as surrogate marker? Cancer Lett 2012;323:88-96.

12. Quabius ES, Haag J, Kuhnel A, Henry H, Hoffmann AS, Gorogh T, et al. Geographical and anatomical influences on human papillomavirus prevalence diversity in head and neck squamous cell carcinoma in Germany. Int J Oncol 2015;46:414-22.

13. Allegra E, Caltabiano R, Amorosi A, Vasquez E, Garozzo A, Puzzo L. Expression of BMI1 and p16 in laryngeal squamous cell carcinoma. Head Neck 2013;35:847-51.

14. Hayry V, Makinen LK, Atula T, Sariola H, Makitie A, Leivo I, et al. Bmi-1 expression predicts prognosis in squamous cell carcinoma of the tongue. Br J Cancer 2010;102:892-7.

15. Jiang L, Li J, Song L. Bmi-1, stem cells and cancer. Acta Biochim Biophys Sin (Shanghai) 2009;41:527-34.

16. Cao L, Bombard J, Cintron K, Sheedy J, Weetall ML, Davis TW. BMI1 as a novel target for drug discovery in cancer. J Cell Biochem 2011;112:2729-41.

17. Ang KK, Harris J, Wheeler R, Weber R, Rosenthal DI, Nguyen-Tan $\mathrm{PF}$, et al. Human papillomavirus and survival of patients with oropharyngeal cancer. N Engl J Med 2010;363:24-35.

18. Chung CH, Zhang Q, Kong CS, Harris J, Fertig EJ, Harari PM, et al. p16 protein expression and human papillomavirus status as prognostic biomarkers of nonoropharyngeal head and neck squamous cell carcinoma. J Clin Oncol 2014;32:3930-8.

19. Molofsky AV, Pardal R, Iwashita T, Park IK, Clarke MF, Morrison SJ. Bmi-1 dependence distinguishes neural stem cell self-renewal from progenitor proliferation. Nature 2003;425:962-7. 